\title{
El Derecho Ambiental y el Tribunal Constitucional del Perú
}

Fernando Calle Hayen*

http://dx.doi.org/10.21503/lex.v10i9.335

Magistrado del Tribunal Constitucional. Doctor en Derecho. Profesor de la USMP, y de la Escuela de Posgrado de la UAP.

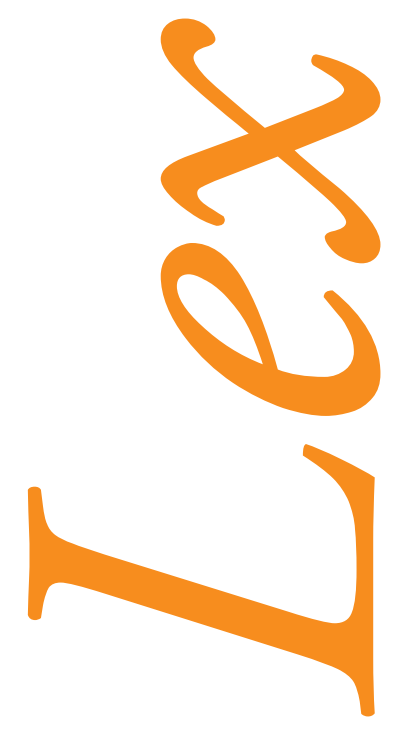




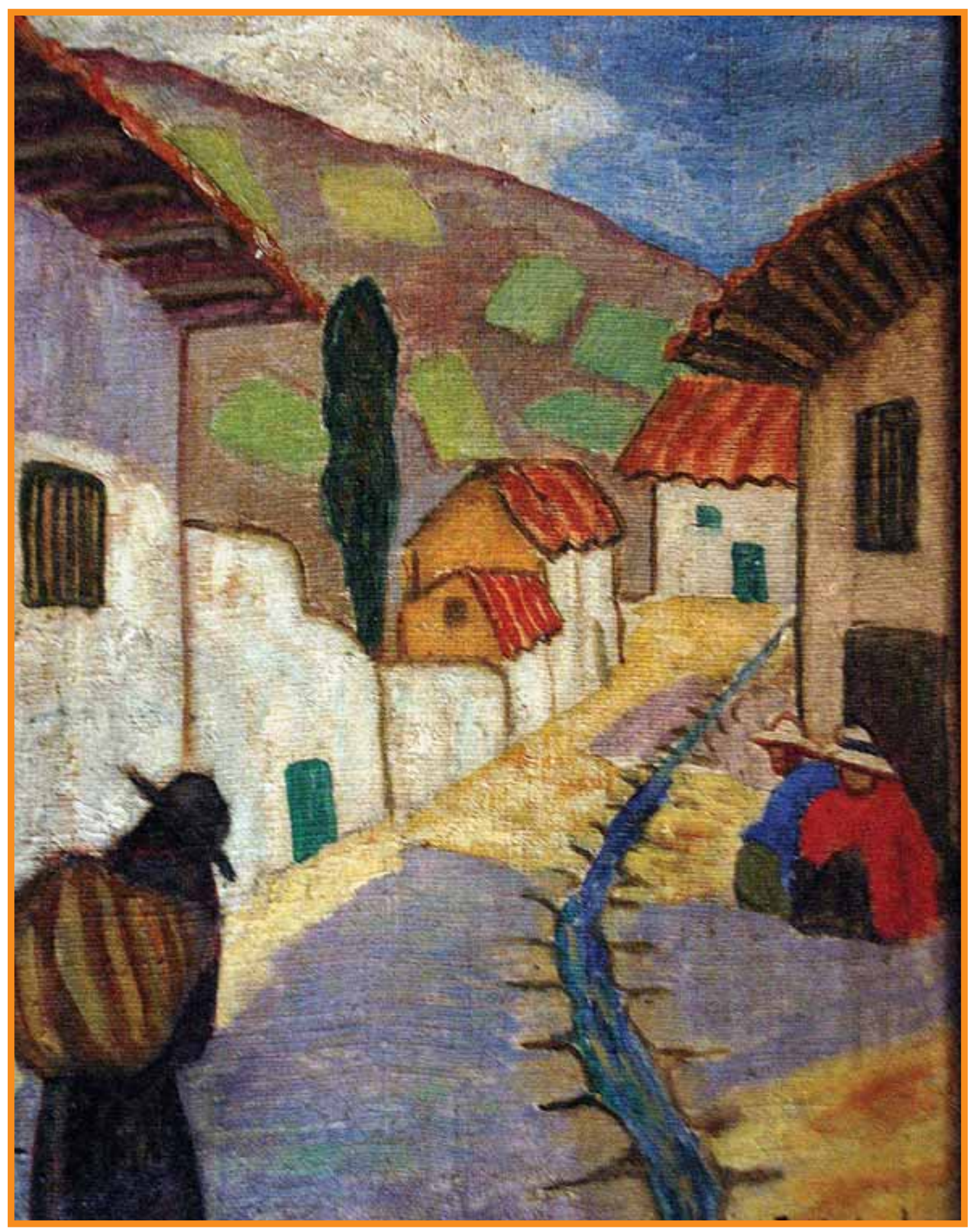

Paisaje. Colección Néstor Benavides. 


\section{El medio ambiente y la Constitución Política del Perú}

Los derechos fundamentales que la Constitución reconoce son efectivamente derechos subjetivos, pero también constituyen la manifestación de un orden material y objetivo de valores constitucionales en los que se sustenta todo el ordenamiento jurídico. Esta última dimensión de los derechos fundamentales se traduce, por un lado, en exigir que las leyes se apliquen conforme a los derechos fundamentales (efecto de irradiación de los derechos en todos los sectores del ordenamiento jurídico), y, por otro, en imponer sobre todos los organismos públicos un deber de tutelar dichos derechos.

Ello no significa que tales derechos solo puedan oponerse a los organismos públicos. El Tribunal Constitucional ha manifestado en múltiples ocasiones que en nuestro sistema constitucional los derechos fundamentales vinculan tanto al Estado como a los particulares. En el caso de autos, la responsabilidad del Estado la comparte, entre otros, con los particulares que promueven actividades que dañan o pueden dañar el medio ambiente.

Es sabido que a través de nuestra historia constitucional (Constituciones de 1979 y de 1993) se ha puesto énfasis en el cuidado del medio ambiente, al punto que las citadas normas fundamentales dedican capítulos exclusivos a la tutela de dichos derechos.

En el artículo No 119 del Capítulo II de la Constitución Política de 1979, se señala que "El Estado evalúa y preserva los recursos naturales. Asimismo fomenta su racional aprovechamiento. Promueve su industrialización para impulsar el desarrollo económico". Asimismo, el numeral 22 del artículo No 1 del Capítulo I de la Constitución de 1993 dice que toda persona tiene derecho "a la paz, a la tranquilidad, al disfrute del tiempo libre y al descanso, así como a gozar de un ambiente equilibrado y adecuado al desarrollo de su vida". Del mismo modo, el artículo 670 del Capítulo II señala que el Estado determina la política nacional del ambiente y promueve el uso sostenible de sus recursos naturales, además de estar obligado a promover la conservación de la diversidad biológica y de las áreas naturales protegidas. 
Dichas normas constitucionales no hacen sino respaldar el desarrollo constitucional de protección al medio ambiente en nuestro país, establecido por los convenios y tratados internacionales que el Perú ha ratificado. El derecho fundamental a un medio ambiente equilibrado y adecuado para el desarrollo de la vida, reconocido en el artículo $2^{\circ}$, inciso 22) de la Constitución, supone el disfrute no de cualquier entorno, sino únicamente el del adecuado para el desarrollo de la persona y de su dignidad. De lo contrario, su goce se vería frustrado y el derecho quedaría, así, carente de contenido. En ese sentido, el derecho al medio ambiente equilibrado y adecuado se encuentra ligado a los derechos fundamentales a la vida y a la salud de las personas, pues por intermedio de él las personas humanas desarrollan su vida en condiciones dignas.

De otra parte, este derecho también se concretiza en el derecho a la preservación de un medio ambiente sano y equilibrado. Esto entraña la obligación ineludible para los poderes públicos de mantener los bienes ambientales en las condiciones adecuadas para su disfrute. Por su parte, los particulares deben proceder de modo similar cuando sus actividades económicas incidan, directa o indirectamente, en el medio ambiente.

De este modo, en el Estado Democrático y Social de Derecho no solo se trata de garantizar la existencia de la persona o cualquiera de los demás derechos que en su condición de ser humano le son reconocidos, sino también de protegerla de los ataques al medio ambiente en el que esa existencia se desenvuelve, a fin de permitir que su vida se desarrolle normalmente en condiciones ambientales aceptables. En este contexto, el derecho a un medio ambiente equilibrado y adecuado debe considerarse como un componente esencial e indispensable para el goce efectivo de los demás derechos fundamentales reconocidos por la Constitución y los tratados internacionales sobre derechos humanos.

De ahí que este derecho, en su dimensión prestacional, imponga al Estado tareas u obligaciones destinadas a conservar el medio ambiente sano y equilibrado, las cuales se traducen, a su vez, en un haz de posibilidades. Desde luego, no solo supone tareas de conservación, sino también de prevención de daños de ese ambiente equilibrado y adecuado para el desarrollo de una vida digna. Dentro de las tareas de prestación que el Estado está llamado a desarrollar, tiene especial relevancia la tarea de prevención y, desde luego, la realización de acciones destinadas a ese fin.

Así, la protección del medio ambiente sano y adecuado no solo es una cuestión de reparación frente a daños ocasionados, sino, y de manera especialmente relevante, de prevención de que aquellos sucedan. De este modo, la protección del medio ambiente puede hacerse efectiva desde la previsión de medidas reactivas que hagan frente a los daños que ya se han producido, pasando por medidas que hagan frente a riesgos conocidos antes de que se 
produzcan (prevención), hasta medidas que prevean y eviten amenazas de daños desconocidos o inciertos (precaución).

El Estado también debe velar por la utilización racional de todos los recursos naturales con el fin de proteger y mejorar la calidad de vida de las personas, y defender y restaurar el medio ambiente dañado, puesto que el desarrollo sostenible involucra un conjunto de instrumentos, entre ellos los jurídicos, que hagan factible el progreso de las próximas generaciones en consonancia con un desarrollo armónico del medio ambiente.

Por tanto, el Estado puede afectar el derecho a un medio ambiente equilibrado y adecuado si, como consecuencia de decisiones normativas o prácticas administrativas, por acción u omisión, en vez de fomentar la conservación del medio ambiente, contribuye a su deterioro o reducción y, en lugar de auspiciar la prevención contra el daño ambiental, descuida y desatiende dicha obligación.

En buena cuenta, el Estado está obligado a velar por la conservación y debida protección del derecho a un medio ambiente equilibrado y adecuado, procurando que el desarrollo económico y social sea compatible con las políticas que buscan salvaguardar las riquezas naturales y el medio ambiente de la Nación.

$Y$ es que la protección del medio ambiente involucra aspectos relacionados con el manejo, uso, aprovechamiento y conservación de los recursos naturales, el equilibrio de los ecosistemas, la protección de la diversidad biológica, la conservación de las áreas de especial importancia ecológica, el desarrollo sostenible y la calidad de vida del hombre en condiciones dignas.

\section{Elderecho a un medio ambiente equilibrado y adecuado como limite a los derechos fundamentales}

En este punto conviene recordar nuestra asentada doctrina sobre la limitación de los derechos fundamentales. En ella se ha afirmado que no existen derechos fundamentales ilimitados, y que, por el contrario, tienen los límites que en relación a los derechos fundamentales establece la Constitución por sí misma en algunos derechos, mientras que en otros derechos el límite deriva de manera mediata o indirecta de tal norma, en cuanto ha de justificarse por la necesidad de proteger o preservar no solo otros derechos fundamentales, sino también otros bienes constitucionales protegidos.

Pues bien, teniendo presente que los derechos fundamentales no son ilimitados, corresponde determinar si la protección de los derechos a un medio ambiente equilibrado y adecuado y a la salud constituye límites legítimos al ejercicio de los derechos al trabajo, a la libertad de empresa y a la libertad de contratación. Ello debido a que la realización de ciertas actividades, como la importación de vehículos usados, motores, partes, piezas y repuestos usados para vehículos de transporte terrestre, el transporte público, la emanación de gases 
tóxicos por parte de las fábricas, implica el cumplimiento de determinados requisitos para que puedan concretarse.

La libertad de empresa consagrada por el artículo $59^{\circ}$ de la Constitución se define como la facultad de poder elegir la organización y efectuar el desarrollo de una unidad de producción de bienes o prestación de servicios, para satisfacer la demanda de los consumidores o usuarios. Consecuentemente, dicha libertad debe ser ejercida con sujeción a la ley, siendo sus limitaciones básicas aquellas que derivan de la seguridad, la salud, la moralidad o la preservación del medio ambiente.

Con relación a la libertad de trabajo consagrada por el artículo 2..$^{\circ}$, inciso 15) de la Constitución, debe subrayarse que ésta debe ser ejercida con sujeción a la ley, siendo sus limitaciones básicas aquellas que derivan de la seguridad, la higiene, la salud, la moralidad o la preservación del medio ambiente.

En tal línea, el artículo 59 de la Constitución establece que el ejercicio de las libertades de trabajo y de empresa "no debe ser lesivo a la moral, ni a la salud, ni a la seguridad públicas, ni al medio ambiente". La protección del medio ambiente tiene entonces una doble dimensión; por un lado, constituye un principio que irradia todo el orden jurídico puesto que es obligación del Estado proteger los recursos naturales de la Nación; y por otro, aparece como el derecho de todas las personas a gozar de un ambiente equilibrado y adecuado para el desarrollo de su vida en condiciones dignas.

\section{La Constitución Ecológica}

Tomando en cuenta doctrina y jurisprudencia constitucional comparada, se ha denominado al conjunto de disposiciones de la Carta Fundamental, referidas a las relaciones entre el individuo, la sociedad y el medio ambiente, Constitución Ecológica (STC 3610-2008-PA/TC, fundamento 33). Así, el artículo 66 de la Constitución establece que los recursos naturales, renovables y no renovables, son patrimonio de la Nación, y que el Estado es soberano en su aprovechamiento. Por su parte, el artículo $67^{\circ}$ de la Constitución dispone que el Estado determine la política nacional del ambiente y promueva el uso sostenible de los recursos naturales. De otro lado, el artículo 68 de la Constitución prescribe: "El Estado está obligado a promover la conservación de la diversidad biológica y de las áreas naturales protegidas". En esa línea, el artículo 690 señala: "El Estado promueve el desarrollo sostenible de la Amazonía”.

El Tribunal Constitucional ha señalado en la STC No 3610-2008-AA la importancia de la Constitución Ecológica. Sobre en particular, el Tribunal entiende que la tutela del medio ambiente se encuentra regulada en nuestra Constitución Ecológica, que no es otra cosa que el conjunto de disposiciones de nuestra Constitución que fijan las relaciones entre el 
individuo, la sociedad y el medio ambiente, tema que ocupa un lugar medular en nuestra Ley Fundamental.

Tal como en su momento fue desarrollado por la Corte Constitucional Colombiana, en criterio que es compartido por este Tribunal, la Constitución Ecológica tiene una triple dimensión:

- Como principio que irradia todo el orden jurídico, puesto que es obligación del Estado proteger las riquezas naturales de la Nación.

- Como derecho de todas las personas a gozar de un ambiente sano, derecho constitucional que es exigible por diversas vías judiciales.

- Como conjunto de obligaciones impuestas a las autoridades y a los particulares, "en su calidad de contribuyentes sociales".

De ahí que se derive un conjunto de acciones que el Estado se compromete a desarrollar y promover, con el fin de preservar y conservar el ambiente frente a las actividades humanas que pudieran afectarlo. Esta política nacional debe permitir el desarrollo integral de todas las generaciones, que tienen el derecho de gozar de un ambiente adecuado para el bienestar de su existencia.

Desarrollando los alcances de los artículos constitucionales referidos, el artículo $9^{\circ}$ de la Ley General del Ambiente, Ley No 28611, establece: "La Política Nacional del Ambiente tiene por objetivo mejorar la calidad de vida de las personas, garantizando la existencia de ecosistemas saludables, viables y funcionales en el largo plazo; y el desarrollo sostenible del país, mediante la prevención, protección y recuperación del ambiente y sus componentes, la conservación y el aprovechamiento sostenible de los recursos naturales, de una manera responsable y congruente con el respeto de los derechos fundamentales de la persona".

El enunciado legal materializa lo determinado en la llamada Constitución Ecológica. Así, en primer lugar, al ser los recursos naturales, in totum, patrimonio de la Nación, su explotación no puede ser separada del interés nacional, por ser una universalidad patrimonial reconocida para los peruanos de las generaciones presentes y futuras. En segundo lugar, los beneficios derivados de su utilización deben alcanzar a la Nación en su conjunto; por ende, se proscribe su exclusivo y particular goce.

Una perspectiva que no debe ser soslayada es la relativa a la consideración de los servicios ambientales que prestan ciertas áreas del territorio de la Nación. Recursos que, en algunos casos, benefician no solo al país, sino también a la región e inclusive a todo el planeta, como por ejemplo la captura de carbono realizada por la selva amazónica. De aquí la relevancia 
de que el Estado asuma la protección de esta riqueza mediante la exhaustiva fiscalización de la explotación de las riquezas ubicadas en estas zonas. Una de las formas de proteger estas riquezas, que además suelen ser ecosistemas frágiles, es la implantación de áreas especialmente protegidas. Con ello se deberá evitar la afectación o disminución de la calidad de los servicios ambientales, como puede ser el caso de la captación y almacenamiento de agua.

\section{Desarrollo sostenible y generaciones futuras}

El uso sostenible de los recursos naturales comporta la utilización de componentes de la diversidad biológica de un modo y a un ritmo que no ocasione la disminución a largo plazo de dicha diversidad, con lo cual se mantienen las posibilidades de esta de satisfacer las necesidades y las aspiraciones de las generaciones actuales y futuras. De igual modo, cuando se explotan recursos no renovables, como los hidrocarburíferos, debe cuidarse no comprometer aquella diversidad biológica.

Al respecto, la Comisión Mundial sobre el Medio Ambiente y Desarrollo de las Naciones Unidas, conocida también como la Comisión Brundtland, emitió un informe en el que definió el desarrollo sostenible como aquel proceso en donde se asegura la satisfacción de las necesidades humanas del presente sin que se ponga en peligro la capacidad de las generaciones futuras para atender sus propias necesidades y que, por ende, involucre la utilización de recursos, la dirección de las inversiones y la orientación de los cambios tecnológicos e institucionales que acrecienten el potencial actual y futuro de los recursos naturales en aras de atender las necesidades y aspiraciones humanas (véase STC 0048-2004-AI/TC).

En dicho informe también se expresa que el "desarrollo sostenible no es un estado concreto, sino un proceso de cambio en donde la explotación de recursos, la dirección de las inversiones, la orientación de los desarrollos tecnológicos y los cambios institucionales deben ser consistentes con el futuro así como con el presente".

Como se aprecia, la perspectiva del desarrollo sostenible busca equilibrar el esquema de la economía social de mercado con el derecho a vivir en un ambiente equilibrado y adecuado. Es una maximización de las ganancias o utilidad frente a la calidad del entorno que sufre el desgaste de la actividad económica. En tal sentido, con el principio de sostenibilidad (artículo $\mathrm{V}$ de la Ley General del Ambiente) se pretende modular esta actividad económica a la preservación del ambiente, el mismo que tendrá que servir de soporte vital también para las generaciones venideras. Así, los derechos de las actuales generaciones no deben ser la ruina de las aspiraciones de las generaciones futuras.

Cabría advertir, no obstante, que no se trata de preservar exclusivamente el legado ambiental, sino también los aspectos relativos al ámbito cultural. Esto significa que nuestra 
deuda con las generaciones futuras no se agota en aspectos ambientales, que si bien forman parte esencial del concepto de desarrollo sostenible, no se agota en él.

En suma, de una interpretación sistemática del artículo $2^{\circ}$, inciso 22 ), y de los artículos $66^{\circ}$, $67^{\circ}, 68^{\circ}$ y $69^{\circ}$ de la Constitución, se concluye que una manifestación concreta del derecho de toda persona a disfrutar de un entorno ambiental idóneo para el desarrollo de su existencia es el reconocimiento de que los recursos naturales -especialmente los no renovables-, en tanto patrimonio de la Nación, deben ser objeto de un aprovechamiento razonable y sostenible, y que los beneficios resultantes de tal aprovechamiento deben ser a favor de la colectividad en general, correspondiendo al Estado el deber de promover las políticas adecuadas a tal efecto.

\section{Política Nacional del Ambiente (STC No 0053-2004-AI/TC)}

El artículo $67^{\circ}$ de la Constitución establece la obligación perentoria del Estado de instituir la politica nacional del ambiente. Ello implica un conjunto de acciones que el Estado se compromete a desarrollar o promover, con el fin de preservar y conservar el ambiente frente a las actividades humanas que pudieran afectarlo.

Esta política nacional -entendida como el conjunto de directivas para la acción orgánica del Estado a favor de la defensa y conservación del ambiente- debe permitir el desarrollo integral de todas las generaciones de peruanos que tienen el derecho de gozar de un ambiente adecuado para el bienestar de su existencia. Esta responsabilidad estatal guarda relación con lo dispuesto en el artículo $2^{\circ}$, inciso 22) de la Constitución, que reconoce el derecho fundamental de toda persona "a gozar de un ambiente equilibrado y adecuado al desarrollo de la vida".

Dicha política debe promover el uso sostenible de los recursos naturales; ergo, debe auspiciar el goce de sus beneficios resguardando el equilibrio dinámico entre el desarrollo socioeconómico de la Nación y la protección y conservación de un disfrute permanente.

El uso sostenible obliga a la tarea de rehabilitar aquellas zonas que hubieren resultado afectadas por actividades humanas destructoras del ambiente y, específicamente, de sus recursos naturales. Por ende, el Estado se encuentra obligado a promover y aceptar únicamente la utilización de tecnologías que garanticen la continuidad y calidad de dichos recursos, evitando que su uso no sostenido los extinga o deprede. Es dentro de ese contexto que el Estado se encuentra obligado a auspiciar la conservación de la diversidad biológica y de las áreas naturales objeto de protección.

En consecuencia, de una interpretación sistemática del artículo $2^{\circ}$, inciso 22) y de los artículos $66^{\circ}$ y $67^{\circ}$ de la Constitución, se concluye que una manifestación concreta del derecho de toda persona a disfrutar de un entorno ambiental idóneo para el desarrollo de su existencia 
es el reconocimiento de que los recursos naturales -especialmente los no renovables- en tanto patrimonio de la Nación, deben ser objeto de un aprovechamiento razonable y sostenible, y que los beneficios resultantes de tal aprovechamiento deben ser a favor de la colectividad en general, correspondiendo al Estado el deber de promover las políticas adecuadas a tal efecto.

\section{El bloque de constitucionalidad para una mejor tutela del derecho a un ambiente equilibrado}

El Tribunal Constitucional ha señalado en su jurisprudencia la importancia del bloque de constitucionalidad para un mejor desarrollo interpretativo de los derechos fundamentales. Es así que en nuestro ordenamiento jurídico, la referencia al parámetro de constitucionalidad o bloque de la constitucionalidad tiene como antecedente inmediato el artículo $22^{\circ}$ de la Ley Orgánica del Tribunal Constitucional, que hoy se ha incorporado en el artículo $79^{\circ}$ del Código Procesal Constitucional como principio de interpretación, cuyo tenor es: “(...) para apreciar la validez constitucional de las normas, el Tribunal Constitucional considerará, además de las normas constitucionales, las leyes que, dentro del marco constitucional, se hayan dictado para determinar la competencia o las atribuciones de los órganos del Estado o el ejercicio de los derechos fundamentales de la persona”.

En estos casos, las infracciones directas a las normas que conforman el parámetro de constitucionalidad determinarán, por consiguiente, afectaciones indirectas a la jerarquía normativa de la Constitución, como lo prevé el artículo $75^{\circ}$ del Código Procesal Constitucional.

En el caso concreto de los derechos fundamentales concernientes al medio ambiente equilibrado y adecuado existen una serie de normas que, aunque no gozan de rango constitucional, ayudan a una mejor interpretación.

Es importante destacar que desde la Reunión de Estocolmo -inclusive antes-, Johannesburgo (Sudáfrica), la Reunión Mundial de Asociaciones de Derecho ambiental en Limoges y La Cumbre de la Tierra, entre otros, muchos Estados viables, sin incorporar el tema en el marco constitucional de sus países, fueron desarrollando, vía jurisprudencial y normas locales, la preservación del medio ambiente y, lo que es más importante, el desarrollo de una conciencia ambiental en sus naciones. Esta es la dirección que fundamentalmente deberíamos seguir, lo que supone la utilización del desarrollo de la ciencia para el cuidado de la vida, orientándonos más que a la norma al cumplimiento de la misma, fortaleciendo la convicción consciente de su necesidad, algo como ciencia con conciencia.

\section{Convenios y tratados}

El Perú ha ratificado varios de los convenios y tratados en tema ambiental, entre los que podemos mencionar: 
- Convención de las Naciones Unidas de Lucha contra la Desertificación en los Países Afectados por Sequía Grave o Desertificación, en particular en África (UNCCD).

- Convención Marco de Naciones Unidas sobre Cambio Climático (UNFCCC).

- Convención Relativa a los Humedales de Importancia Internacional especialmente como Hábitat de Aves Acuáticas (RAMSAR).

- Convención sobre el Comercio Internacional de las Especies Amenazadas de Fauna y Flora Silvestre (CITES).

- Convenio sobre Diversidad Biológica (CDB).

- Convenio de Basilea sobre el Control de los Movimientos Transfronterizos de los Desechos Peligrosos y su Eliminación.

- Convenio de Rotterdam sobre el Procedimiento de Consentimiento previo Fundamentado aplicable a ciertos Plaguicidas y Productos Químicos Peligrosos objeto de Comercio Internacional (PIC).

- Convenio de Estocolmo sobre Contaminantes Orgánicos Persistentes (POPs).

- Convenio de Viena para la Protección de la Capa de Ozono.

- Protocolo de Montreal relativo a Sustancias Agotadoras de la Capa de Ozono.

- Convención sobre la Conservación de las Especies Migratorias de Animales (CMS).

- Convenio para el Manejo y Conservación de la Vicuña.

- Convención Internacional para la Regulación de la Caza de las Ballenas (Comisión Ballenera Internacional).

- Convención sobre la Conservación de los Recursos Marinos Vivos Antárticos (CCAMLR).

- Convención sobre la Protección del Patrimonio Mundial, Cultural y Natural.

- Cumbre Mundial sobre el Desarrollo Sostenible (Johannesburgo, 2002).

- Proceso Cumbre de las Américas.

- Conferencia de las Naciones Unidas sobre Medio Ambiente y Desarrollo (Río, 1992). 


\section{Jurisprudencia constitucional}

a) Caso Nextel (STC No 4223-2006-AA/TC)

Mediante sentencia No 4223-2006-AA/TC, el Tribunal Constitucional se pronunció acerca del caso Nextel, donde el demandante exigía vivir en un medio ambiente equilibrado, solicitando que se ordene el inmediato desmantelamiento de la antena de telecomunicaciones y demás equipos instalados por la empresa en un Centro Comunal, pues su permanencia constituía una grave vulneración del derecho a la paz, a la tranquilidad y a gozar de un ambiente equilibrado y adecuado al desarrollo de la vida, así como del derecho a la salud de los pobladores de dicha urbanización.

El Colegiado puso bajo análisis varios principios establecidos en la jurisprudencia constitucional en cuanto al vínculo existente entre las actividades económicas y el derecho a un ambiente equilibrado y adecuado al desarrollo de la vida: (1) el principio de desarrollo sostenible o sustentable; (2) el principio de conservación, en cuyo mérito se busca mantener en estado óptimo los bienes ambientales; (3) el principio de prevención, que supone resguardar los bienes ambientales de cualquier peligro que pueda afectar su existencia; (4) el principio de restauración, referido al saneamiento y recuperación de los bienes ambientales deteriorados; (4) el principio de mejora, en cuya virtud se busca maximizar los beneficios de los bienes ambientales en pro del disfrute humano; (5) el principio precautorio, que comporta adoptar medidas de cautela y reserva cuando existan incertidumbre científica e indicios de amenaza sobre la real dimensión de los efectos de las actividades humanas sobre el ambiente; y (6) el principio de compensación, que implica la creación de mecanismos de reparación por la explotación de los recursos no renovables (STC 0048-2004-PI/TC).

Si bien el TC no dispuso el desmantelamiento de la antena de la referida empresa, puesto que hubiera sido irrazonable y desproporcionada ya que no siempre la prohibición absoluta de determinada actividad es la única vía para alcanzar determinado grado de protección que depende de cada caso y puede ser alcanzado mediante la reducción de la exposición al riesgo, con el establecimiento de mayores controles y la imposición de ciertas limitaciones-, sí dispuso la realización de diversas mediciones. Así, de los informes solicitados por el TC se concluyó que no existía riesgo de exposición radioeléctrica, lo cual no constituía razón para exceptuar la realización de las mediciones correspondiente de exposición radioeléctrica de la población, garantizando así la no afectación de los derechos fundamentales al medio ambiente y a la salud.

b) Caso Repsol-Cordillera Escalera (STC No 3343-2007-PA/TC)

En la sentencia recaída en el Exp. No 03343-2007-PA/TC se declaró fundada la demanda 
de amparo y se ordenó la suspensión de la última fase de la etapa de exploración y la etapa de explotación dentro del Área de Conservación Regional denominada Cordillera Escalera hasta que no contara con un plan maestro, pudiendo reiniciarse tal actividad una vez que este hubiese sido elaborado y se estableciera la compatibilidad entre la actividad de exploración y explotación y los objetivos del Área de Conservación Regional Cordillera Escalera.

El fallo fue debido a que se comprobó la inexistencia del Plan Maestro, responsabilidad de las autoridades estatales competentes y no de las empresas emplazadas. Por esta razón, y con el propósito de emitir una decisión que denotara un adecuado equilibrio entre la debida protección del medio ambiente y el aprovechamiento razonable de los recursos naturales, el Tribunal solo suspendió las etapas referidas mientras no se contara con el Plan Maestro.

Ciertamente, la sentencia obedeció también a que el Área de Conservación Regional Cordillera Escalera es un área relevante no solo para el país en conjunto sino en especial para la región San Martín, en tanto constituye una importante fuente de agua, facilita la captura de carbono, presenta una gran biodiversidad, etc.

Otra razón del fallo es que dicha área tiene como objetivos generales los siguientes: a) conservar y proteger los recursos naturales y la diversidad biológica de los ecosistemas frágiles que se encuentran en la Cordillera Escalera, y b) asegurar la comunidad de los procesos biológicos en los ecosistemas del área propuesta (artículo $2^{\circ}$ del Decreto Supremo No 0452005-AG). Además, obedece al hecho de que las Áreas Naturales Protegidas por el Estado tienen por finalidad, entre otras cosas, asegurar la continuidad de los procesos ecológicos y evolutivos, mantener la biodiversidad y preservar la base de recursos, incluyendo los genéticos, que permitan desarrollar opciones para mejorar los sistemas productivos y encontrar adaptaciones frente a eventuales cambios climáticos.

\section{c) Caso Autos Usados}

En este caso, el TC declaró infundada la demanda interpuesta, ya que consideró que el Decreto Supremo No 017-2005-MTC constituye un límite legítimo al ejercicio de los derechos a la libertad de trabajo y empresa, pues el establecimiento de requisitos para la importación de vehículos usados, así como de motores, partes, piezas y repuestos usados para vehículos de transporte terrestre, tiene como fin constitucional la protección de los derechos al medio ambiente equilibrado y adecuado y a la salud, y además porque la protección del medio ambiente impone un tratamiento cuyo propósito es mejorar progresivamente las condiciones de vida de las personas, toda vez que la creciente degradación del medio ambiente pone en peligro potencial la propia base de la vida.

Este Colegiado tuvo presente, al momento de evaluar la sentencia, que los derechos fundamentales no son ilimitados, de modo que corresponde determinar si la protección de 
los derechos a un medio ambiente equilibrado y adecuado y a la salud constituyen límites legítimos al ejercicio de los derechos al trabajo, a la libertad de empresa y a la libertad de contratación. Así, consideró que la importación de vehículos usados, motores, partes, piezas y repuestos usados para vehículos de transporte terrestre constituye una actividad económica que está sujeta al cumplimiento de determinados requisitos para que pueda realizarse, los mismos que en consideración de la demandante son restricciones inconstitucionales. 